\title{
Patterns of Tumor Contrast Enhancement Predict the Prognosis of Anaplastic Gliomas with IDH1 Mutation
}

\author{
Y.Y. Wang, K. Wang, S.W. Li, J.F. Wang, J. Ma, T. Jiang, and J.P. Dai
}

\begin{abstract}
BACKGROUND AND PURPOSE: It is proposed that isocitrate dehydrogenase 1 (IDHT) mutation predicts the outcome in patients with high-grade glioma. In addition, contrast enhancement on preoperative MR imaging reflects tumor biologic features. Patients with anaplastic glioma with the IDHI mutation were evaluated by using MR imaging to determine whether tumor enhancement is a prognostic factor and can be used to predict survival.
\end{abstract}

MATERIALS AND METHODS: A cohort of 216 patients with histologically confirmed anaplastic glioma was reviewed retrospectively. Tumor contrast-enhancement patterns were classified on the basis of preoperative $\mathrm{Tl}$ contrast MR images. Tumor IDHI status was examined by using RNA sequencing. We used univariate analysis and the multivariate Cox model to evaluate the prognostic value of the IDHI mutation and tumor contrast-enhancement pattern for progression-free survival and overall survival.

RESULTS: In all 216 patients, IDH1 mutation was associated with longer progression-free survival $(P=.004$, hazard ratio $=0.439)$ and overall survival $(P=.002$, hazard ratio $=0.406)$. For patients with $I D H 1$ mutant anaplastic glioma, the absence of contrast enhancement was associated with longer progression-free survival $(P=.038$, hazard ratio $=0.473)$ and overall survival $(P=.043$, hazard ratio $=0.436)$. Furthermore, we were able to stratify the progression-free survival and overall survival of patients with IDHI mutation by using the tumor contrast-enhancement patterns ( $P=.022$ and 0.029 , respectively; log-rank).

CONCLUSIONS: Tumor enhancement on postcontrast MR imaging is a valuable prognostic factor for patients with anaplastic glioma and $I D H 1$ mutation. Furthermore, the contrast-enhancement patterns could potentially be used to stratify the survival outcome of such patients.

ABBREVIATIONS: $\mathrm{AG}=$ anaplastic glioma; GTR = gross total resection; $\angle \mathrm{GTR}=$ residual tumor; $\mathrm{HR}=$ hazard ratio; IDHI = isocitrate dehydrogenase 7 ; $\mathrm{KPS}=$ Karnofsky Performance Status Scale; PFS = progression-free survival; OS = overall survival

A naplastic gliomas (AGs), classified as World Health Organization grade III, are aggressive brain tumors. They exhibit morphometric heterogeneity on radiologic imaging, and their clinical course varies substantially so that some patients succumb

Received January 26, 2015; accepted after revision March 21.

From the Departments of Neurosurgery (Y.Y.W., J.F.W., T.J.) and Neuroradiology (K.W., S.W.L., J.M., J.P.D.), Beijing Tian Tan Hospital, and Beijing Neurosurgical Institute (Y.Y.W., T.J., J.P.D.), Capital Medical University, Beijing, China; and Center for Brain Tumor (T.J.), Beijing Institute for Brain Disorders, Beijing, China.

Yinyan Wang and Kai Wang contributed equally to this article.

The authors declare that they have no conflict of interest.

This work was supported by the National High Technology Research and Development Program No. 2011 CB707804 and No. 2015 CB755500 (T.J.) and the National Natural Science Foundation of China No. 81271541 (J.P.D.).

Please address correspondence to Tao Jiang, PhD, Beijing Neurosurgical Institute, Capital Medical University, 6, Tiantanxili, Beijing, 100050, China; e-mail: taojiang1964@163.com; and Jianping Dai, PhD, Beijing Neurosurgical Institute, Capital Medical University, 6, Tiantanxili, Beijing, 100050, China; e-mail: djpbeijing@hotmail.com; @taojiang1964

- Indicates open access to non-subscribers at www.ajnr.org

http://dx.doi.org/10.3174/ajnr.A4407 to progressive disease within weeks while others survive for a decade or more. The clinical characteristics, radiologic features, genetic changes, and extent of resection all play important roles in determining the prognosis of patients with AG ${ }^{1-9}$ Interaction and synergy may exist among these factors.

The presence of contrast enhancement on MR images, which is based on pathophysiologic changes indicating the destruction of the blood-brain barrier, is considered a specific radiologic feature of high-grade gliomas. Previous studies have revealed the prognostic role of contrast enhancement in patients with AG. ${ }^{5,8,10-12}$ Additionally, radiologic features such as enhancement and multifocality correlate with the molecular characteristics of malignant glioma. ${ }^{13}$

Mutation in the isocitrate dehydrogenase 1 (IDH1) gene at $\mathrm{R} 132$ is an important molecular event and plays a significant role in gliomagenesis. This genetic change is detected in approximately $50 \%-70 \%$ of anaplastic astrocytomas ${ }^{1,14,15}$ and in $70 \%$ of anaplastic oligodendrogliomas. ${ }^{14-16}$ Furthermore, the presence of 
IDH1 mutations distinguishes tumors with markedly different clinical presentations, concurrent molecular genetic alterations, and overall natural history. ${ }^{1}$ For patients with AG, the occurrence of IDH1 mutation is associated with gross total resection (GTR) and longer overall survival (OS). ${ }^{17}$

Previous studies have demonstrated the correlation between IDH1 status and the radiologic features of glioma, in that tumors with $I D H 1$ mutation are more likely to be larger, and cyst, edema, and contrast enhancement are present. ${ }^{18}$ However, investigations of the interactive and synergistic role of $I D H 1$ mutation and tumor contrast enhancement in predicting the survival of patients with AG are rare. By classifying tumor contrast enhancement according to the patterns observed, patients with anaplastic glioma with IDH1 mutation were evaluated by using MR imaging to determine whether tumor enhancement is a prognostic factor and can be used to predict outcome survival stratification.

\section{MATERIALS AND METHODS \\ Patients}

In total, 216 adult patients diagnosed with AG who had undergone surgical treatment at our institution from February 2007 to June 2010 were reviewed retrospectively. Patients were included on the basis of the following criteria: 1) age 18 years or older, 2) presurgical structural MR imaging scan available (T1-weighted, T2-weighted, postcontrast T1-weighted), 3) pathology-confirmed AG based on the modified World Health Organization grading system, and 4) no previous diagnosis of any brain tumor. The histopathologic diagnosis was evaluated and confirmed by 2 independent neuropathologists blinded to the patients' clinical and radiologic information. According to the Response Assessment in Neuro-Oncology criteria, ${ }^{19}$ GTR was defined as no visible contrast enhancement on postoperative MR images within 72 hours after the operation in contrast-enhanced tumors or absence of all abnormal hyperintense changes on preoperative MR images for tumors not demonstrating contrast enhancement. In this study, resections that were not GTR were considered residual tumor ( $<$ GTR). The overall follow-up duration was 85 months, which spanned March 2007 to April 2014. This study was approved by our institutional review board, and written consent was obtained from all enrolled patients.

\section{Imaging Acquisition}

MR imaging was performed on Trio 3T scanners (Siemens, Erlangen, Germany). It typically included axial T1-weighted (TR, 450 ms; TE, $15 \mathrm{~ms}$; section thickness, $5 \mathrm{~mm}$ ), T2-weighted fast spinecho (TR, $6000 \mathrm{~ms}$; TE, $140 \mathrm{~ms}$; section thickness, $5 \mathrm{~mm}$ ), and Gd-DTPA injection- enhanced (Beijing Beilu Pharmaceutical, Beijing, China; $0.1 \mathrm{mmol} / \mathrm{kg}$ ) axial T1-weighted images (TR, 450 ms; TE, $15 \mathrm{~ms}$; section thickness, $5 \mathrm{~mm}$ ), with a 24-cm FOV and $256 \times 256$ matrix size. Postcontrast images were acquired immediately following injection of the contrast agent. The interval between contrast injection and the beginning of the contrast-enhanced T1-weighted image acquisition was maintained between 75 and 85 seconds. Postoperative MR images for determining the extent of resection were obtained within 72 hours after resection, and the radiologic parameters were maintained in accordance with the preoperative scans.

\section{Identification of Imaging Features}

Tumor contrast enhancement was assessed by 2 experienced neuroradiologists (Q.C. and X.C., who have 14 and 12 years of experience, respectively, in diagnosis using brain $\mathrm{MR}$ imaging) blinded to the patient clinical information. In cases in which the types of enhancement identified by the first 2 neuroradiologists were inconsistent, a third senior neuroradiologist (J.M., 25 years of experience in brain disease diagnosis) re-examined the images and determined the image to be used. "Contrast enhancement" was defined as newly emerged unequivocal increased signal intensity on the T1-weighted image following intravenous contrast administration compared with noncontrast T1 images. "Nonenhancement" was defined as no apparent hyperintensity on postcontrast T1-weighted images. Three contrast-enhancement patterns were identified on the basis of the size and morphologic features of the largest enhanced area on contrast-enhanced MR images regardless of whether it was single or multifocal: nodular, with the largest focal diameter of $\leq 1.5 \mathrm{~cm}$; patchy, tumors with a maximum diameter of enhancement of $>1.5 \mathrm{~cm}$; and ringlike, cystic necrosis with peripheral enhancement (Fig 1). Multifocal tumor enhancement was defined as $>1$ area of tumor enhancement separated from the others on the postcontrast T1-weighted image.

\section{DNA Sequencing for IDH1 Mutation}

IDH1 mutation was determined by using DNA pyrosequencing, which we have described previously. ${ }^{20}$ Briefly, a QIAamp DNA Mini Kit (QIAGEN, Hilden, Germany) was used to isolate genomic DNA from frozen tumor tissue samples. We then analyzed the genomic region spanning the wild type R132 of IDH1 by using pyrophosphate sequencing with $5^{\prime}$-GCTTGTGAGTGGATGGGTAAAAC-3' and 5' -biotin-TTGCCAACATGACTTACTTGATC-3' primers. Duplicate polymerase chain reaction analyses were performed in $40-\mu \mathrm{L}$ reaction volumes containing $1-\mu \mathrm{L}$ of each $10-\mu \mathrm{mol} / \mathrm{L}$ forward and reverse primer, $4 \mu \mathrm{L}$ of $10 \times$ buffer, $3.21 \mu \mathrm{L}$ of $2.5-\mathrm{mmol} / \mathrm{L}$ deoxynucleotide triphosphates, 2.5-U HotStar Taq (Takara, Shiga, Japan), and $2 \mu \mathrm{L}$ of $10 \mu \mathrm{mol} / \mathrm{L}$ DNA. The polymerase chain reaction conditions were as follows: $95^{\circ} \mathrm{C}$ for 3 minutes; 50 cycles of $95^{\circ} \mathrm{C}$ for 15 seconds, $56^{\circ} \mathrm{C}$ for 20 seconds, $72^{\circ} \mathrm{C}$ for 30 seconds; and $72^{\circ} \mathrm{C}$ for 5 minutes (Applied Biosystems GeneAmp PCR System 9700; Applied Biosystems, Foster City, California). Single-stranded DNA was purified from the polymerase chain reaction products and pyrosequenced with a PyroMark Q96 ID System (QIAGEN) by using a $5^{\prime}$-TGGATGGGTAAAACCT-3' primer and an EpiTect Bisulfite Kit (QIAGEN).

\section{Statistical Analysis}

We used the $\chi^{2}$ test for categoric variables to compare each clinical and imaging feature between the IDH1 mutant and wild type groups. The agreement between judgments of the enhancement patterns assessed by the 2 radiologists was evaluated by using the $\kappa$ consistency test. A $\kappa$ value of $\geq 0.81,0.61-0.80$, and $\leq 0.60$ was considered excellent, good, and poor agreement, respectively. Additionally, log-rank analysis of Kaplan-Meier data was performed to compare the progression-free survival (PFS) and OS of the cohort. Factors that were significant $(P<.05)$ in univariate anal- 


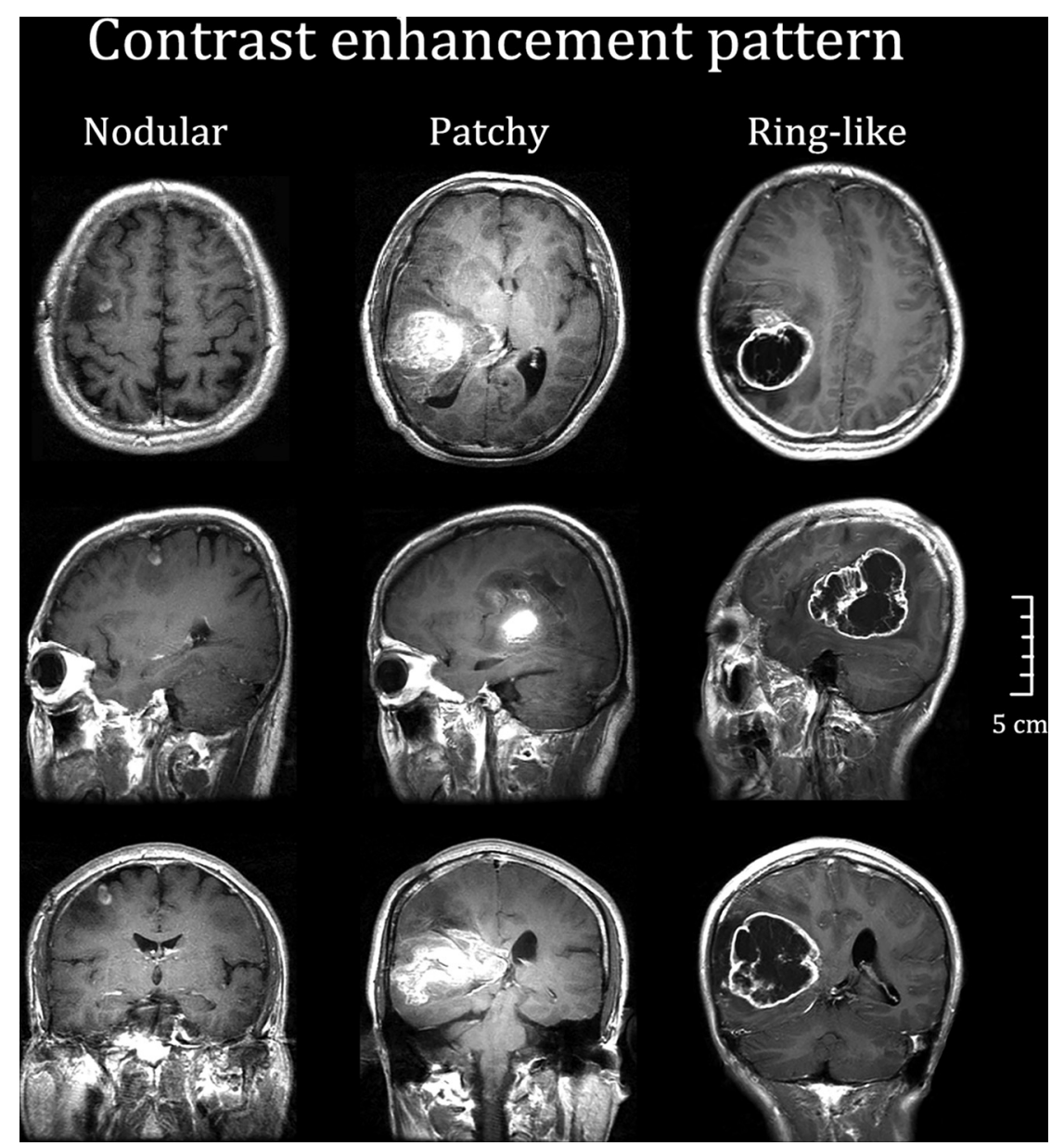

FIG 1. Tumor contrast-enhancement patterns in AG. Postcontrast T1-weighted images depict the nodular (largest focal diameter of $\leq 1.5 \mathrm{~cm}$ ), patchy (largest focal diameter of $>1.5 \mathrm{~cm}$ ), and ringlike (cystic necrosis with peripheral enhancement) enhancement patterns.

Table 1: IDH1 mutation status of patients with AG

\begin{tabular}{|c|c|c|c|c|}
\hline \multirow[b]{2}{*}{ Characteristics } & \multicolumn{3}{|c|}{ IDH1 Status } & \multirow[b]{2}{*}{$P$ Value $^{\mathrm{a}}$} \\
\hline & Total $(n=216)$ & Mutant $(n=84)$ & Wild Type $(n=132)$ & \\
\hline \multicolumn{5}{|l|}{ Age (yr) } \\
\hline Median (range) & $44(18-87)$ & 43 (18-71) & 45 (18-87) & \\
\hline 50 or older $/ 50$ or younger & 77:139 & 23:61 & $54: 78$ & .043 \\
\hline \multicolumn{5}{|l|}{ Sex } \\
\hline Male/female & 135:81 & $49: 35$ & $86 / 46$ & .313 \\
\hline \multicolumn{5}{|l|}{ KPS } \\
\hline$\geq 80 /<80$ & $181 / 35$ & $78 / 6$ & $103 / 29$ & .004 \\
\hline Contrast enhancement & & & & \\
\hline Yes/no & $173 / 43$ & $57 / 27$ & $116 / 16$ & $<.001$ \\
\hline \multicolumn{5}{|l|}{ Pattern of enhancement } \\
\hline Nodular/patchy/ringlike & $26 / 62 / 85$ & $9 / 14 / 34$ & $17 / 48 / 51$ & .084 \\
\hline \multicolumn{5}{|l|}{ Extent of resection } \\
\hline GTR/<GTR & $123 / 93$ & $56 / 28$ & $67 / 65$ & .021 \\
\hline \multicolumn{5}{|l|}{ Histopathology } \\
\hline $\mathrm{AA} / \mathrm{AO} / \mathrm{AOA}$ & $57 / 44 / 115$ & $16 / 20 / 48$ & $41 / 24 / 67$ & .135 \\
\hline
\end{tabular}

ysis were entered into multivariate survival analysis on the basis of the Cox proportional hazard ratio (HR) model. To identify the prognostic value of IDH1 status and tumor contrast-enhancement pattern in patients according to their interactive effects, we subdivided patients into 4 subgroups according to these 2 indicators. The respective prognostic values of the tumor contrast-en- hancement pattern of the IDH1 mutant and wild type groups were evaluated.

\section{RESULTS}

Patient Characteristics

The clinical and radiologic data of the 216 patients with AG were reviewed (Table 1). Among these patients, $57(26.4 \%)$ had anaplastic astrocytoma, $44(20.4 \%)$ had anaplastic oligodendroglioma, and 115 (53.2\%) had anaplastic oligoastrocytoma. Age at diagnosis, preoperative Karnofsky Performance Status Scale (KPS), and extent of resection were significantly different between patients with mutant and wild type IDH1 $\left(P<.001, \chi^{2}\right.$ test $)$. A total of $123(56.9 \%)$ patients underwent GTR, and $93(43.1 \%)$ patients had residual tumor.

\section{Association between IDH1} Mutation and Tumor Enhancement There was post-T1 contrast enhancement in $173(80.1 \%)$ tumors. Patients with $I D H 1$ mutation were less likely to have MR imaging tumor enhancement than patients with wild type IDH1 (67.9\% versus $87.9 \%, P<.001)$. In addition, tumor contrast-enhancement patterns were identified in the AGs with enhancement. The $\kappa$ value for the agreement of judgment of enhancement patterns between the 2 evaluators was 0.96 $(P=.012)$. Enhancement was nodular in $26(15.0 \%)$ cases, patchy in 62 $(35.9 \%)$ cases, and ringlike in 85 (49.1\%) cases (Table 1). However, there was no significant difference between the proportion of contrast-enhancement patterns between tumors from patients with mutant and wild type IDH1 $(P=.084)($ Fig 2$)$.

\section{Association between Surgical Resection and Tumor Enhancement} Of the tumors with contrast enhancement, those with ringlike enhancement patterns were more likely to undergo GTR than tumors without ringlike enhancement patterns, but the difference was not statistically significant $(59.7 \%$ versus $46.9 \%, P=.113)$. Notably, patients with mutant IDH1 and tumors with ringlike enhancement patterns were also more likely to undergo GTR than patients with mutant $I D H 1$ and tumors without ringlike enhancement patterns $(65.3 \%$ versus $37.5 \%, P=.004)$. However, in patients with wild type $I D H 1$, GTR between tumors with and without 
ringlike enhancement patterns was not significantly different (47.8\% versus $66.7 \%, P=.157)$.

\section{Progression-Free Survival}

There was tumor recurrence in 165 (76.4\%) patients during the follow-up period; the median PFS was 16.9 months (range, 3.1-82.8 months). Univariate analysis showed that patients with mutant $I D H 1$ had significantly longer PFS than patients with wild type IDH1 ( $P=.002, \log$-rank). Additionally, age at diagnosis $(P<.001)$, preoperative KPS $(P=.004)$, and GTR $(P=.001)$ were significant prognostic factors for PFS (Table 2). In multivariate Cox regression analysis, wild type IDH1 $(P=.004, \mathrm{HR}=2.277 ; 95 \%$ confidence interval, $1.303-3.968)$, preoperative KPS $<80(P=.015, \mathrm{HR}=2.158$; 95\% CI, $1.179-$ $3.471)$, age at diagnosis older than 50 years $(P=.018, \mathrm{HR}=$ 1.857; 95\% CI, 1.111-3.106), and $<$ GTR $(P=.028$, HR $=$ $1.598 ; 95 \% \mathrm{CI}, 1.053-2.597)$ were associated with poor PFS (Table 3).

\section{Overall Survival}

At the time of analysis, 69 patients (whose follow-up data were available) were still alive; the median follow-up period was 22.9 months (range, 3.3-86.4 months). In univariate analysis, IDH1 status $(P=.004)$, age at diagnosis $(P=.007)$, preoperative KPS $(P=.002)$, and extent of resection $(P<.001)$ were prognostic factors of OS (Table 2). These 4 factors remained significant in the multivariate Cox proportional hazards analysis: Wild type $I D H 1$

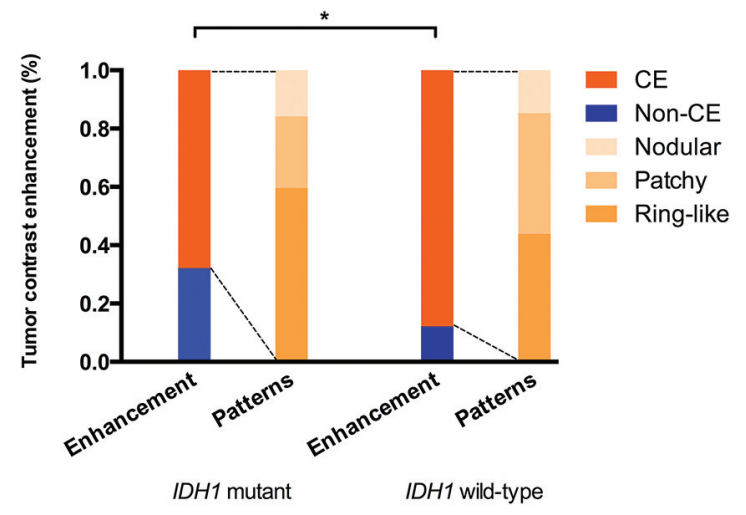

FIG 2. Constitution of tumor contrast enhancements between AG accompanied by mutant or wild type IDHI. The difference in contrastenhancement rate (asterisk) between tumors from patients with mutant and wild type $I D H I$ was significant $(P<.001)$. There was no significant difference in enhancement-pattern distribution between tumors from patients with mutant and wild type IDHI ( $P=.135)$. CE indicates contrast enhancement.

Table 2: Univariate analysis of survival outcomes for patients with AG

\begin{tabular}{|c|c|c|c|c|c|c|}
\hline \multirow[b]{2}{*}{ Characteristic } & \multicolumn{3}{|c|}{ PFS } & \multicolumn{3}{|c|}{ OS } \\
\hline & $P$ Value & HR & $95 \% \mathrm{Cl}$ & $P$ Value & HR & $95 \% \mathrm{Cl}$ \\
\hline Age 50 yr or older & $<.001$ & 1.813 & $1.318-2.494$ & .007 & 1.729 & $1.134-2.421$ \\
\hline Sex (male) & .215 & 0.873 & $0.695-1.064$ & .415 & 0.914 & $0.612-1.283$ \\
\hline Preoperative KPS $<80$ & .004 & 2.603 & $1.154-3.548$ & .002 & 2.872 & $1.270-3.341$ \\
\hline Enhancement & .570 & 1.200 & $0.641-2.247$ & .625 & 1.187 & $0.596-2.365$ \\
\hline Enhancement pattern & .150 & 1.107 & $0.964-1.271$ & .247 & 1.902 & $0.941-1.266$ \\
\hline$<$ GTR/GTR & .001 & 1.734 & $1.237-2.429$ & $<.001$ & 1.926 & $1.346-2.758$ \\
\hline Histopathology & .086 & 0.855 & $0.715-1.022$ & .054 & 0.789 & $0.654-1.002$ \\
\hline IDHI wild type & .002 & 2.364 & $1.362-4.098$ & .004 & 2.688 & $1.231-4.717$ \\
\hline
\end{tabular}

$(P=.002, \mathrm{HR}=2.463 ; 95 \% \mathrm{CI}, 1.389-4.386)$, age at diagnosis 50 years and older $(P=.016, \mathrm{HR}=1.431 ; 95 \% \mathrm{CI}, 1.342-2.434)$, preoperative KPS $<80(P=.026, \mathrm{HR}=1.836$; $95 \% \mathrm{CI}, 1.087-$ $3.402)$, and $<$ GTR $(P=.023, \mathrm{HR}=1.488$; 95\% CI, $1.210-2.432)$ were poor prognostic factors for OS (Table 3 ).

\section{Prognostic Role of Tumor Contrast-Enhancement Pattern for Patients with Mutant IDH1}

In the mutant IDH1 group, patients with contrast-enhanced tumors had significantly shorter PFS (median PFS, 15.9 months; range, 2.6-76.8 months) than those with nonenhanced tumors (median PFS, 26.3 months; range, $2.7-80.3$ months $)(P=.038$, log-rank) (Fig 3). Furthermore, the tumor contrast-enhancement patterns allowed us to stratify the PFS of patients with mutant IDH1. Patients with nodular enhancement patterns had significantly longer PFS (median, 23.8 months) than those with patchy (median, 17.6 months) $(P=.042$, log-rank) or ringlike enhancement patterns (median, 14.7 months $)(P=.010$, log-rank). There were no significant differences between the PFS of patients with patchy or ringlike enhancement patterns $(P=.273$, log-rank) (Fig $4)$. In comparison, the tumor contrast enhancement patterns had no prognostic value in patients with wild type IDH1 $(P=.896$, log-rank).

Patients were subdivided into 4 groups according to IDH1 status and tumor contrast enhancement. Notably, among the 4 groups, patients with mutant $I D H 1$ and nonenhanced tumor had significantly longer OS than patients in the other groups $(P=$ .043 , Fig 3 ). Tumor contrast-enhancement pattern played a prognostic role in the OS of patients with mutant IDH1. In the mutant IDH1 group, patients with nodular enhancement patterns had significantly longer OS (median, 31.8 months) than those with patchy (median, 27.1 months $)(P=.025$, log-rank) and ringlike enhancement patterns (median, 20.3 months) $(P=.012$, logrank). There were no significant differences between the OS of patients with patchy enhancement patterns and those with ringlike enhancement patterns $(P=.441, \log$-rank) (Fig 4$)$. In comparison, tumor contrast enhancement patterns were not a prognostic factor in patients with wild type $I D H 1$ ( $P=.842$, log-rank).

\section{Prognostic Role of Extent of Resection for Patients with Mutant IDH1 and Contrast Enhancement}

In the mutant $I D H 1$ and contrast-enhancement groups, patients with GTR had significantly longer PFS (median, 19.2 months; range, 2.1-75.8 months) than those with $<$ GTR (median, 13.0 months; range, 3.0-73.7 months) $(P=.018$, log-rank). Similarly, for patients with mutant $I D H 1$ and contrast-enhanced tumors, GTR also predicted significantly longer OS than $<$ GTR $(P=.030$, 
log-rank). Nevertheless, GTR did not have prognostic power for PFS and OS in the wild type IDH1 and contrast-enhancement groups (PFS, $P=.224$; OS, $P=.141$, respectively; log-rank).

\section{DISCUSSION}

We combined clinical, radiologic, and specific genetic characteristics to investigate the prognostic factors for a large cohort of patients with AG, demonstrating that IDH1 mutation was an independent prognostic factor for patients with AG. Furthermore, the tumor contrast-enhancement pattern identified from postcontrast MR imaging was associated with the survival outcomes of patients with mutant IDH1. To our knowledge, this is the first

Table 3: Multivariate analysis of survival outcomes

\begin{tabular}{lccc}
\hline \multicolumn{1}{c}{ Predictor } & $P$ Value $^{\mathrm{a}}$ & HR & 95\% CI \\
\hline PFS & & & \\
$\quad$ Age 50 yr or older & .018 & 1.857 & $1.111-3.106$ \\
$\quad$ Preoperative KPS $<80$ & .015 & 2.158 & $1.179-3.471$ \\
$\quad$ <GTR & .028 & 1.598 & $1.053-2.597$ \\
$\quad$ IDH7 wild type & .004 & 2.277 & $1.303-3.968$ \\
OS & & & \\
$\quad$ Age 50 yr or older & .016 & 1.431 & $1.342-2.434$ \\
$\quad$ Preoperative KPS $<80$ & .026 & 1.836 & $1.087-3.402$ \\
$\quad$ <GTR & .023 & 1.488 & $1.210-2.432$ \\
$\quad$ IDH7 wild-type & .002 & 2.463 & $1.389-4.386$ \\
\hline
\end{tabular}

${ }^{a}$ Cox proportional hazard regression analyses. A $P$ value of .05 denoted significance. investigation of the prognostic role of combined IDH1 mutation and tumor contrast-enhancement pattern for predicting survival in patients with AG.

Previous studies have demonstrated the impact of IDH1 mutation on the clinical prognosis of patients with malignant glioma. ${ }^{1}$ In patients with AG, it has been demonstrated that IDH1 mutation is a good prognostic marker and potential stratification factor for anaplastic astrocytoma and anaplastic oligodendroglioma. ${ }^{20,21}$ Consistent with these findings, the present study showed that patients with mutant $I D H 1$ had significantly longer PFS and OS than those with wild type IDH1. The better prognosis of patients with mutant IDH1 may be partly attributed to the effect of $I D H 1$ interaction with other clinical characteristics. First, IDH1 mutation is frequent in diffuse low-grade gliomas but rare in primary glioblastomas, and the survival outcome of patients with low-grade glioma is generally better than that of patients with glioblastoma. ${ }^{1,14,15,22}$ This variance of IDH1 mutation incidence between low- and high-grade gliomas may contribute to the association of $I D H 1$ mutation with good prognosis. In addition, IDH1 mutation is more common in younger than elder patients. ${ }^{23}$ Because age is a widely reported significant prognostic factor, ${ }^{2,4,6,18,24}$ the prognosis of patients with mutant IDH1 might be good.

In this study, the frequency of IDH1 mutation between the 2
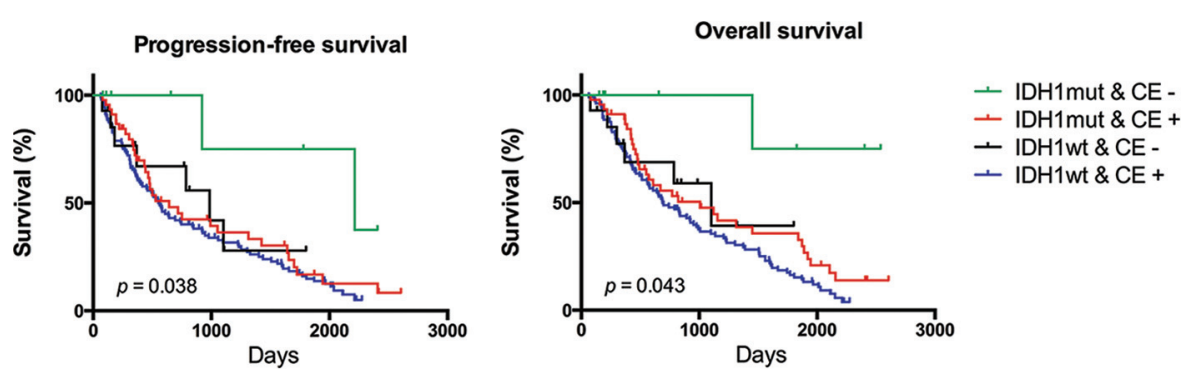

FIG 3. Kaplan-Meier plots of the series of 216 patients with AG showing the association between the PFS and OS according to combined IDHI status and tumor contrast enhancement. Mutant IDHI with no contrast enhancement predicts better survival (PFS, $P=.038 ;$ OS, $P=.043$ ). mut indicates mutant; wt, wild type.
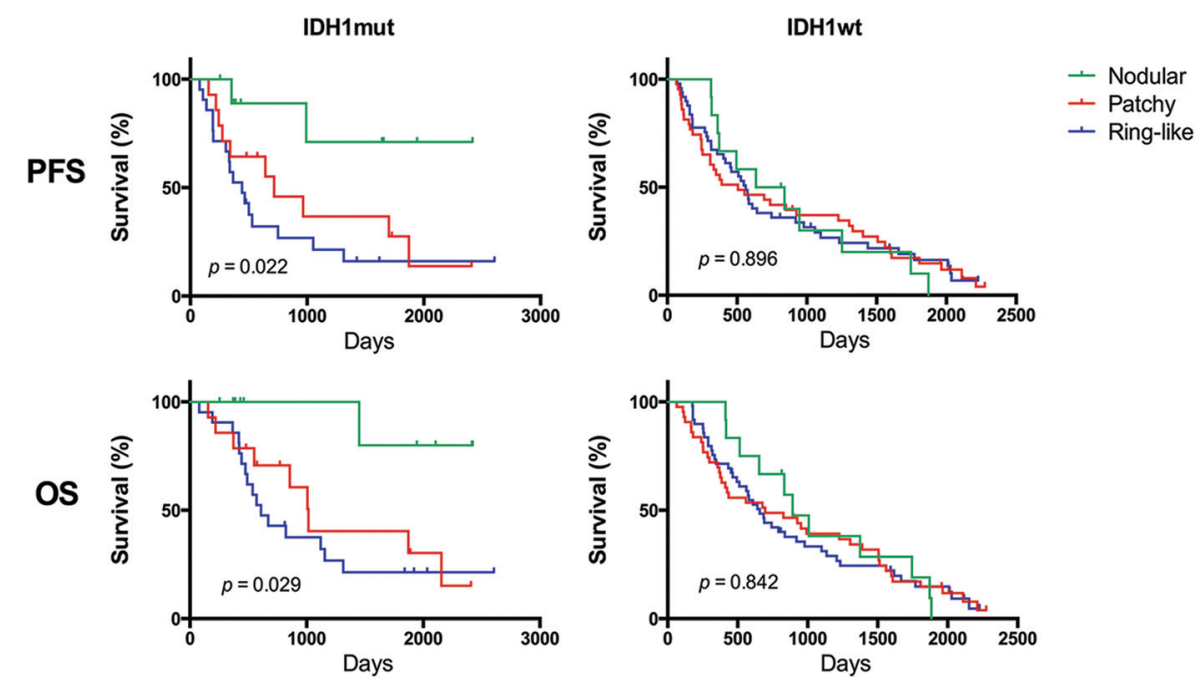

FIG 4. Kaplan-Meier plots showing that the tumor contrast enhancement pattern enabled stratification of the PFS and OS of patients with mutant IDH7 (PFS, $P=.022$; OS, $P=.029$ ). Meanwhile, Kaplan-Meier plots show that the tumor contrast-enhancement pattern did not enable stratification of the PFS and OS of patients with wild type IDHI (PFS, $P=.896 ; \mathrm{OS}, P=.842$ ). 
age groups (50 years and older and younger than 50 years of age at diagnosis) was marginally significantly different $(P=.043)$; these results agreed with the previous ones. Furthermore, this and a previous study ${ }^{25}$ found that GTR was more likely to be achieved in AG with mutant $I D H 1$ than in AG with wild type IDH1. There was a higher rate of ringlike enhancement patterns in patients with mutant $I D H 1$; in addition, these patients were more likely to undergo GTR than patients whose tumors did not have ringlike enhancement patterns, which could also have contributed to the difference in survival outcome between the 2 subgroups.

On the other hand, previous studies have suggested that the good prognosis of patients with mutant $I D H 1$ is primarily due to the less aggressive biologic behavior of tumors with mutant IDH1 compared with tumors with wild type IDH1. ${ }^{17,19,26}$ Because IDH1 mutation is considered an early genetic event in tumorigenesis and may drive other genetic changes in tumor cells, tumors accompanied by IDH1 mutation may consequently have different genetic characteristics compared with tumors unaccompanied by the mutation, which may lead to their varied biologic features. The intrinsic difference in the tumor biologic features may explain why the IDH1 mutation, though associated with other clinical characteristics, was an independent prognostic factor for patients with AG. Other than IDH1 mutation, however, the histopathologic subtypes in the present study did not have prognostic value, indicating that the variety of tumor components could not predict survival for patients with AG.

It has been reported that the radiologic features of glioma are associated with $I D H 1$ mutation. A recent study showed that $I D H 1$ mutation status in glioblastoma can be predicted from the radiologic features derived from MR images. ${ }^{18}$ The study identified 4 subjective tumor characteristics observable on MR images: tumor size, contrast enhancement, and the presence or absence of cyst and satellite lesions, which were associated with IDH1 status; these characteristics predicted the presence of IDH1 mutation with $94 \%$ accuracy (by receiver operating characteristic analysis). Another study demonstrated that IDH1-mutated gliomas were predominantly located in a single lobe and were more likely to have a unilateral growth pattern, sharp tumor margin, homogeneous signal intensity, and less contrast enhancement on MR imaging. ${ }^{27}$ In the present study, we also found that tumor contrast enhancement was associated with IDH1 status. Tumors accompanied by mutant IDH1 were less likely to show contrast enhancement on MR images compared with tumors without IDH1 mutation. Most interesting, although there was no significant difference in the contrast-enhancement patterns between tumors accompanied by mutant or wild type $I D H 1$, multivariate Cox analysis identified the tumor contrast-enhancement pattern as an independent prognostic factor in patients with mutant IDH1. This result implies that the tumor contrast-enhancement pattern may be a particularly important factor reflecting the biologic features of tumors in the presence of IDH1 mutation.

The prognostic value of IDH1 status and tumor contrast enhancement was determined in patients with AG, considering their interactive effects. Notably, among the 4 classifications based on the 2 indicators, patients with mutant IDH1 and nonenhanced tumor had significantly longer PFS and OS (Fig 3) than patients who did not; there was no difference in survival time among the other 3 groups. This indicates that IDH1 mutation and the absence of contrast enhancement may have synergistic effects in reflecting tumor malignancy and predicting survival outcome.

Most interesting, tumor contrast-enhancement patterns were identified as a prognostic marker that could be used to stratify PFS and OS only for patients with mutant $I D H 1$, but not for patients with wild type $I D H 1$. Specifically, patients with mutant $I D H 1$ with nodular enhancement patterns had longer PFS and OS than those with mutant $I D H 1$ with patchy or ringlike enhancement patterns $(P=.022)$. Tumor contrast enhancement reflects the degree of destruction of the blood-brain barrier, which is induced by tumor cell invasion. Therefore, the tumor contrast-enhancement pattern may be strongly associated with the biologic features of a tumor. A small area of tumor enhancement (nodular pattern) possibly indicates lower grade malignancy compared with a relatively large area of tumor enhancement (patchy or ringlike pattern), which explains the present findings. Why the tumor contrast-enhancement pattern plays a more important role in predicting the survival of patients with AG with mutant IDH1 compared with those with wild-type IDH1 remains to be investigated.

In addition, the extent of resection in the enhanced tumors could be used to stratify PFS and OS for patients with mutant IDH1 - that is, patients with GTR had longer PFS and OS than patients with $<$ GTR; but this finding was not true for patients with wild type IDH1. As discussed above, there was a higher rate of ringlike enhancement pattern in the tumors of patients with mutant IDH1. Compared with tumors with nodular or patchy enhancement patterns, tumors with ringlike enhancement patterns had a relatively clear border on postcontrast T1-weighted images, which might facilitate surgical resection of the tumor bulk. On the other hand, IDH1 mutation is common in diffuse low-grade gliomas and is more common in younger patients; these findings indicate less aggressive behavior and contribute to effective resection of most of the tumor in patients with mutant $I D H 1$.

Our study has some limitations. First, we retrospectively enrolled patients from a single institution; therefore, the prognostic role of tumor contrast-enhancement patterns and IDH1 mutation requires confirmation by a prospective multicenter investigation. Second, due to the limited number of patients and the similar distribution of IDH1 mutation in the 3 anaplastic glioma subtypes, we did not separate patients by pathology for further discussion. Third, due to the relatively suboptimal timing of the postoperative scans, the potential presence of granulation tissue is a confounder in the MR imaging. The interval from contrast agent administration to image acquisition could also have influenced the level of enhancement. Although the study was carefully controlled, a slight discrepancy in the interval between contrast injection and scanning may still have been present among individuals. Future studies should investigate the association between the radiologic characteristics and survival of patients with tumors with other gene mutations.

\section{CONCLUSIONS}

We retrospectively reviewed 216 patients with AG and identified IDH1 mutation as a significant prognostic factor. In this study, we found that the tumor contrast-enhancement patterns were asso- 
ciated with the survival outcome of patients with mutant IDH1. Our results imply that there may be a synergistic effect between radiologic morphology and the genetic features of a tumor in determining prognosis, and this effect should be considered in future investigations.

\section{ACKNOWLEDGMENTS}

We thank Drs Q. Chen and X. Chen for their effort in tumor segmentation.

Disclosures: Jun Ma-UNRELATED: Grants/Grants Pending: National Natural Science Foundation of China (No. 81371610). ${ }^{*}$ Tao Jiang-RELATED: Grant: National Ministry of Science and Technology, ${ }^{*}$ Comments: National High Technology Research and Development Program No. 2011 CB707804 and No. 2015CB755500; Jianping DaiRELATED: Grant: China Ministry of Science and Technology, ${ }^{*}$ Comments: This work was supported by the National Natural Science Foundation of China No. 81271541. *Money paid to the institution.

\section{REFERENCES}

1. Hartmann C, Hentschel B, Wick W, et al. Patients with IDH1 wild type anaplastic astrocytomas exhibit worse prognosis than IDH1mutated glioblastomas, and IDH1 mutation status accounts for the unfavorable prognostic effect of higher age: implications for classification of gliomas. Acta Neuropathol 2010;120:707-18 CrossRef Medline

2. Paleologos NA, Merrell RT. Anaplastic glioma. Curr Treat Options Neurol 2012;14:381-90 CrossRef Medline

3. DeAngelis LM. Anaplastic glioma: how to prognosticate outcome and choose a treatment strategy. [corrected]. J Clin Oncol 2009;27: 5861-62 CrossRef Medline

4. Reyes-Botero G, Dehais C, Idbaih A, et al; POLA Network. Contrast enhancement in 1p/19q-codeleted anaplastic oligodendrogliomas is associated with 9p loss, genomic instability, and angiogenic gene expression. Neuro Oncol 2014;16:662-70 CrossRef Medline

5. Laigle-Donadey F, Martin-Duverneuil N, Lejeune J, et al. Correlations between molecular profile and radiologic pattern in oligodendroglial tumors. Neurology 2004;63:2360-62 CrossRef Medline

6. Parkinson JF, Afaghi V, Payne CA, et al. The impact of molecular and clinical factors on patient outcome in oligodendroglioma from $\mathbf{2 0}$ years' experience at a single centre. J Clin Neurosci 2011;18:329-33 CrossRef Medline

7. Shirai K, Suzuki Y, Okamoto M, et al. Influence of histological subtype on survival after combined therapy of surgery and radiation in WHO grade 3 glioma. J Radiat Res 2010;51:589-94 CrossRef Medline

8. Tortosa A, Viñolas N, Villa S, et al. Prognostic implication of clinical, radiologic, and pathologic features in patients with anaplastic gliomas. Cancer 2003;97:1063-71 CrossRef Medline

9. Ramirez C, Bowman C, Maurage CA, et al. Loss of 1p, 19q, and 10q heterozygosity prospectively predicts prognosis of oligodendroglial tumors-towards individualized tumor treatment? Neuro Oncol 2010;12:490-99 CrossRef Medline

10. Chaichana KL, Kosztowski T, Niranjan A, et al. Prognostic significance of contrast-enhancing anaplastic astrocytomas in adults. J Neurosurg 2010;113:286-92 CrossRef Medline

11. Matar E, Cook RJ, Fowler AR, et al. Post-contrast enhancement as a clinical indicator of prognosis in patients with anaplastic astrocytoma. J Clin Neurosci 2010;17:993-96 CrossRef Medline
12. Mangla R, Ginat DT, Kamalian S, et al. Correlation between progression free survival and dynamic susceptibility contrast MRI perfusion in WHO grade III glioma subtypes. J Neurooncol 2014;116: 325-31 CrossRef Medline

13. Pope WB, Sayre J, Perlina A, et al. MR imaging correlates of survival in patients with high-grade gliomas. AJNR Am J Neuroradiol 2005; 26:2466-74 Medline

14. Balss J, Meyer J, Mueller W, et al. Analysis of the IDH1 codon 132 mutation in brain tumors. Acta Neuropathol 2008;116:597-602 CrossRef Medline

15. Hartmann C, Meyer J, Balss J, et al. Type and frequency of IDH1 and IDH2 mutations are related to astrocytic and oligodendroglial differentiation and age: a study of 1,010 diffuse gliomas. Acta Neuropathol 2009;118:469-74 CrossRef Medline

16. Jiao Y, Killela PJ, Reitman ZJ, et al. Frequent ATRX, CIC, FUBP1 and IDH1 mutations refine the classification of malignant gliomas. Oncotarget 2012;3:709-22 Medline

17. Ren X, Cui X, Lin S, et al. Co-deletion of chromosome $1 \mathrm{p} / 19 \mathrm{q}$ and IDH1/2 mutation in glioma subsets of brain tumors in Chinese patients. PLoS One 2012; 7:e32764 CrossRef Medline

18. Carrillo JA, Lai A, Nghiemphu PL, et al. Relationship between tumor enhancement, edema, IDH1 mutational status, MGMT promoter methylation, and survival in glioblastoma. AJNR Am J Neuroradiol 2012;33:1349-55 CrossRef Medline

19. Vogelbaum MA, Jost S, Aghi MK, et al. Application of novel response/progression measures for surgically delivered therapies for gliomas: Response Assessment in Neuro-Oncology (RANO) Working Group. Neurosurgery 2012;70:234-43; discussion 243-44 CrossRef Medline

20. Frenel JS, Leux C, Loussouarn D, et al. Combining two biomarkers, IDH1/2 mutations and $1 \mathrm{p} / 19 \mathrm{q}$ codeletion, to stratify anaplastic oligodendroglioma in three groups: a single-center experience. J Neurooncol 2013;114:85-91 CrossRef Medline

21. Zhang CB, Bao ZS, Wang HJ, et al. Correlation of IDH1/2 mutation with clinicopathologic factors and prognosis in anaplastic gliomas: a report of 203 patients from China. J Cancer Res Clin Oncol 2014; 140:45-51 CrossRef Medline

22. Sanson M, Marie $\mathrm{Y}$, Paris S, et al. Isocitrate dehydrogenase $\mathbf{1}$ codon 132 mutation is an important prognostic biomarker in gliomas. J Clin Oncol 2009;27:4150-54 CrossRef Medline

23. Dunn GP, Andronesi OC, Cahill DP. From genomics to the clinic: biological and translational insights of mutant IDH1/2 in glioma. Neurosurg Focus 2013;34:E2 CrossRef Medline

24. Youland RS, Brown PD, Giannini C, et al. Adult low-grade glioma: 19-year experience at a single institution. Am J Clin Oncol 2013;36: 612-19 CrossRef Medline

25. Beiko J, Suki D, Hess KR, et al. IDH1 mutant malignant astrocytomas are more amenable to surgical resection and have a survival benefit associated with maximal surgical resection. Neuro Oncol 2014;16:81-91 CrossRef Medline

26. Stancheva G, Goranova T, Laleva M, et al. IDH1/IDH2 but not TP53 mutations predict prognosis in Bulgarian glioblastoma patients. BioMed Res Int 2014;2014:654727 CrossRef Medline

27. Qi S, Yu L, Li H, et al. Isocitrate dehydrogenase mutation is associated with tumor location and magnetic resonance imaging characteristics in astrocytic neoplasms. Oncol Lett 2014;7:1895-902 CrossRef Medline 